\title{
EFEITO DA ESTIMULAÇÃO ACÚSTICA CONTRALATERAL NAS MEDIDAS TEMPORAIS DAS EMISSÕES OTOACÚSTICAS
}

\author{
Effect of contralateral acoustic stimulation in temporal measures \\ of otoacoustic emissions
}

\author{
Vanessa Nogueira Leme ${ }^{(1)}$, Renata Mota Mamede Carvallo ${ }^{(2)}$
}

\begin{abstract}
RESUMO
Objetivo: comparar o nível de resposta e o tempo de latência das emissões otoacústicas sem e com apresentação de ruído contralateral. Métodos: foram avaliados 30 indivíduos, com idade entre 18 e 30 anos, sem queixas auditivas e com presença de emissões otoacústicas por produto de distorção. Analisou-se o nível de resposta e latência das emissões otoacústicas por meio do programa Latencygram, com e sem apresentação de ruído contralateral tipo "White noise". Resultados: houve diminuição significante nos níveis de resposta das emissões otoacústicas com apresentação de ruído contralateral, confirmando o efeito de supressão relacionado com a ação do sistema eferente; contudo, não houve diferença significante nos valores de latência das emissões otoacústicas. Conclusão: 0 sistema eferente atua apenas na modulação dos níveis de resposta das emissões otoacústicas, contudo não interfere nas medidas temporais das mesmas.
\end{abstract}

DESCRITORES: Emissões Otoacústicas Espontâneas; Tempo de Reação; Estimulação Acústica

\section{INTRODUÇÃO}

O fenômeno das emissões otoacústicas (EOA) diz respeito à liberação de energia sonora na cóclea, a qual se propaga na orelha média até alcançar o meato acústico externo. As EOA são pesquisadas como forma de avaliação da integridade funcional da cóclea, mais especificamente das células ciliadas externas ${ }^{1}$.

As EOA por Produto de Distorção (EOAPD) consistem em uma resposta intermodulada e distorcida, produzida pelo órgão de Corti em resposta a dois estímulos de tom puro, simultâneos ${ }^{2}$. As EOAPD podem fornecer informações sobre frequências específicas com relação ao estado funcional da cóclea e dar uma noção da configuração audiométrica de um indivíduo ${ }^{3}$.

O tempo de latência das EOAPD foi definido como o tempo em que o estímulo acústico leva para

(1) Fonoaudióloga; Especialização em Audiologia Clínica pela Faculdade de Medicina da Universidade de São Paulo; Mestranda em Ciências da Reabilitação pela Faculdade de Medicina da Universidade de São Paulo.

(2) Fonoaudióloga; Professora Livre-Docente do curso de Fonoaudiologia da Faculdade de Medicina da Universidade de São Paulo, FMUSP, São Paulo, SP. alcançar o local gerador da emissão otoacústica dentro da cóclea, ser analisado e retornar ao meato acústico externo para ser captado ${ }^{4}$.

A medida da latência das EOAPD ainda não é largamente conhecida e utilizada na prática clínica, contudo seu estudo pode ser útil na análise da maturação coclear, uma vez que o tempo de latência é determinado pela progressão da onda viajante ${ }^{5}$.

A via auditiva eferente mais largamente estudada é o sistema olivococlear, o qual liga o complexo olivar superior à cóclea ${ }^{6}$. Este sistema é constituído pelos feixes olivococlear medial e lateral, os quais possuem diferenças anatômicas e fisiológicas e coordenam a função interdependente das duas orelhas ${ }^{7}$.

O sistema auditivo eferente medial participa na modulação das emissões otoacústicas, na sensitividade auditiva, na detecção do sinal em ruído, na seletividade de frequências e na função de proteção coclear contra superestimulação acústica ${ }^{7}$.

Portanto, por meio da ação do sistema olivococlear eferente, o nível de resposta das EOA, em humanos com audição normal, pode ser reduzido com a aplicação de estimulação acústica por meio de ruído competitivo ipsilateral, contralateral ou binaural, fenômeno este conhecido como supressão das EOA, que é determinado pela subtração dos níveis 
de resposta na condição com ruído competitivo da condição sem ruído competitivo ${ }^{7-13}$. No entanto, nos indivíduos portadores de doenças retrococleares não deveria ser observada esta redução, sendo que a pesquisa da supressão das EOA pode vir a ser útil no diagnóstico de lesões retrococleares ${ }^{8}$.

Os estudos citados anteriormente confirmam o fenômeno da supressão das EOA, ou seja, a apresentação de ruído contralateral reduz o nível de resposta das EOA, efeito relacionado com o sistema olivococlear medial. Entretanto, ainda é pouco descrito na literatura qual o efeito desta estimulação acústica nas medidas temporais das EOA.

Desta forma, o objetivo do presente estudo é comparar o tempo de latência das EOAPD sem e com a apresentação de ruído contralateral em adultos com a audição normal. Foi pesquisada também a influência do sexo, lado de orelha e frequência sonora na magnitude de resposta e na latência das EOAPD.

\section{MÉTODOS}

O estudo, prospectivo e transversal, foi desenvolvido no Laboratório de Investigação Fonoaudiológica em Audição Humana do Departamento de Fisioterapia, Fonoaudiologia e Terapia Ocupacional da Faculdade de Medicina da Universidade de São Paulo.

A casuística foi composta por 30 indivíduos (totalizando 60 orelhas), sendo 15 do sexo masculino e 15 do feminino, com idade entre 18 e 30 anos e média de idade de 23 anos.

Os critérios de inclusão na amostra deste estudo foram: faixa etária entre 18 e 30 anos, limiares auditivos dentro da normalidade bilateralmente, presença de emissões otoacústicas por produto de distorção dentro dos parâmetros de normalidade bilateralmente. Os critérios de exclusão foram: presença de queixas relacionadas à audição (perda auditiva, otalgia, zumbido etc.), queixas relacionadas a doenças neurológicas, desordens pigmentares do tipo vitiligo, uso contínuo de medicamentos ototóxicos, exposição a ruído ocupacional.

O material utilizado foi: otoscópio da marca Heine, equipamento "ILO 92 Otodynamics Analyser Version 5.6", acoplado a um computador IBM-Pentium II; imitanciômetro modelo AZ-7, para a estimulação acústica contralateral por ruído do tipo "white noise".

Inicialmente os sujeitos foram informados sobre os objetivos e procedimentos da pesquisa e, uma vez concordando em sua participação, assinaram Termo de Consentimento Livre e Esclarecido.

Foi realizada anamnese para obtenção de dados de identificação e presença ou ausência de queixas relacionadas à audição, para a observância dos critérios de inclusão e exclusão da pesquisa.

Em seguida, foi realizada a pesquisa das EOAPD. Para participar da pesquisa, o sujeito deveria apresentar EOAPD em níveis de resposta dentro dos parâmetros de normalidade. A pesquisa das EOAPD foi realizada por meio do teste "Latency-Gram" (LT-gram), com estímulo apresentado na intensidade de 70 dB NPS para L1 e L2, analisando-se passos de dois pontos por oitava. A razão f2/f1 utilizada foi 1,219. Foram consideradas presentes as EOAPD que apresentaram relação sinal/ruído dentro ou acima da faixa de normalidade e $3 \mathrm{~dB}$ NPS ou mais, acima do segundo desviopadrão do ruído de fundo. A varredura de frequências abrangia a faixa de f2 de 1001 a $6384 \mathrm{~Hz}$ e foi apresentada automaticamente pelo equipamento, sendo aceitas no mínimo três e no máximo oito varreduras para obtenção de respostas.

Caso o sujeito atendesse aos critérios de inclusão na pesquisa, era realizado o protocolo do presente estudo, pesquisando o tempo de latência das emissões otoacústicas por produto de distorção, utilizando-se o programa "Latency-gram" (LT-grama), inicialmente sem a apresentação de ruído contralateral e posteriormente com a apresentação de ruído contralateral do tipo "white noise", na intensidade de $60 \mathrm{db}$ NPS (calibrado através de medidor de nível de pressão sonora "Realistic" Escala A). Foi apresentado o mesmo número de varreduras de frequências para as duas situações, sendo no mínimo três e no máximo oito varreduras, para evitar efeito do número de varreduras sobre a medida do nível de resposta das EOA. Os valores de latência das respostas das EOAPD foram captados a partir do método de mudança de fases que consiste na variação de frequências para encontrar as latências. Mantém-se uma frequência primária (f1) e a outra frequência secundária (†2) é a variável. A pesquisa do tempo de latência das respostas das EOAPD foi realizada a passos de dois pontos por oitava, analisando-se as mesmas frequências coletadas na pesquisa do nível de resposta das EOAPD (1001, 2515, 3174, 4004, 5042 e $6384 \mathrm{~Hz}$ ).

Esta pesquisa foi aprovada pela Comissão de Pesquisa do Departamento sob o número de protocolo 396/04.

Os dados desta pesquisa foram submetidos à análise estatística descritiva e inferencial, sendo adotado o nível de significância de $5 \%$ ( $p \leq 0,05)$. Para esta análise, foram utilizados os testes estatísticos não paramétricos de Wilcoxon, Mann-Whitney, Friedman, T-Student, Correlação de Spearman e Teste de Correlação. 


\section{RESULTADOS}

Comparando-se os lados de orelha, quanto aos níveis de amplitude de resposta, não foi observada diferença estatisticamente significante, exceto na frequência de $1 \mathrm{kHz}(p=0,031)$, em que a orelha direita apresentou maiores níveis de resposta na condição sem ruído e maior efeito de supressão das EOAPD.

Quanto à latência de resposta, não foi observada diferença estatisticamente significante entre os lados das orelhas, exceto na frequência de $5 \mathrm{kHz}$ $(p=0,012)$ na condição sem ruído.

A comparação, entre os sexos, da média dos níveis de respostas das EOAPD nas condições sem e com ruído contralateral encontra-se ilustrada na Figura 1.

Observou-se que o sexo feminino apresentou níveis de resposta significantemente maiores nas frequências de $2(p=0,001), 3(p=0,026), 4(p=0,036)$, $5(p=0,009)$ e $6(p=0,044) \mathrm{kHz}$ na situação sem ruído. Contudo, não foi observada diferença estatisticamente significante entre os sexos no efeito de supressão das EOAPD (diferença entre nível de resposta na situação sem ruído e com ruído).

Quanto aos valores de latência das EOAPD, houve uma tendência do sexo masculino apresentar maiores valores de latências, contudo, observou-se diferença estatisticamente significante entre os sexos apenas para as frequências de $5(p=0,006)$ e $6(p=0,023) \mathrm{kHz}$, na condição sem ruído. Assim como observado anteriormente para o efeito de supressão dos níveis de resposta das EOAPD, para os valores de latência das EOAPD também não foi encontrada diferença estatisticamente significante entre os sexos nas diferenças dos valores de latência nas situações sem e com ruído.

Comparando-se os valores de latência das EOAPD entre as frequências sonoras, os resultados demonstraram diferença estatisticamente significante $(p<0,05)$ entre todas as frequências analisadas, sendo que os valores de latência diminuem com o aumento da frequência sonora, conforme ilustra a Figura 2.

Comparando-se as respostas obtidas na condição sem e com apresentação de ruído contralateral, observou-se uma diminuição estatisticamente significante dos níveis de resposta das EOAPD nas frequências de $1,5 \mathrm{kHz}(p=0,045) ; 3 \mathrm{kHz}(p=0,017)$ e $5 \mathrm{kHz}(\mathrm{p}=0,038)$ quando se apresentou estimulação acústica por meio de ruído contralateral. Entretanto, não foi observada diferença estatisticamente significante nos valores de tempo de latência das EOAPD comparando-se as situações sem e com apresentação de ruído contralateral, em nenhuma das frequências pesquisadas, conforme ilustra a Figura 3.

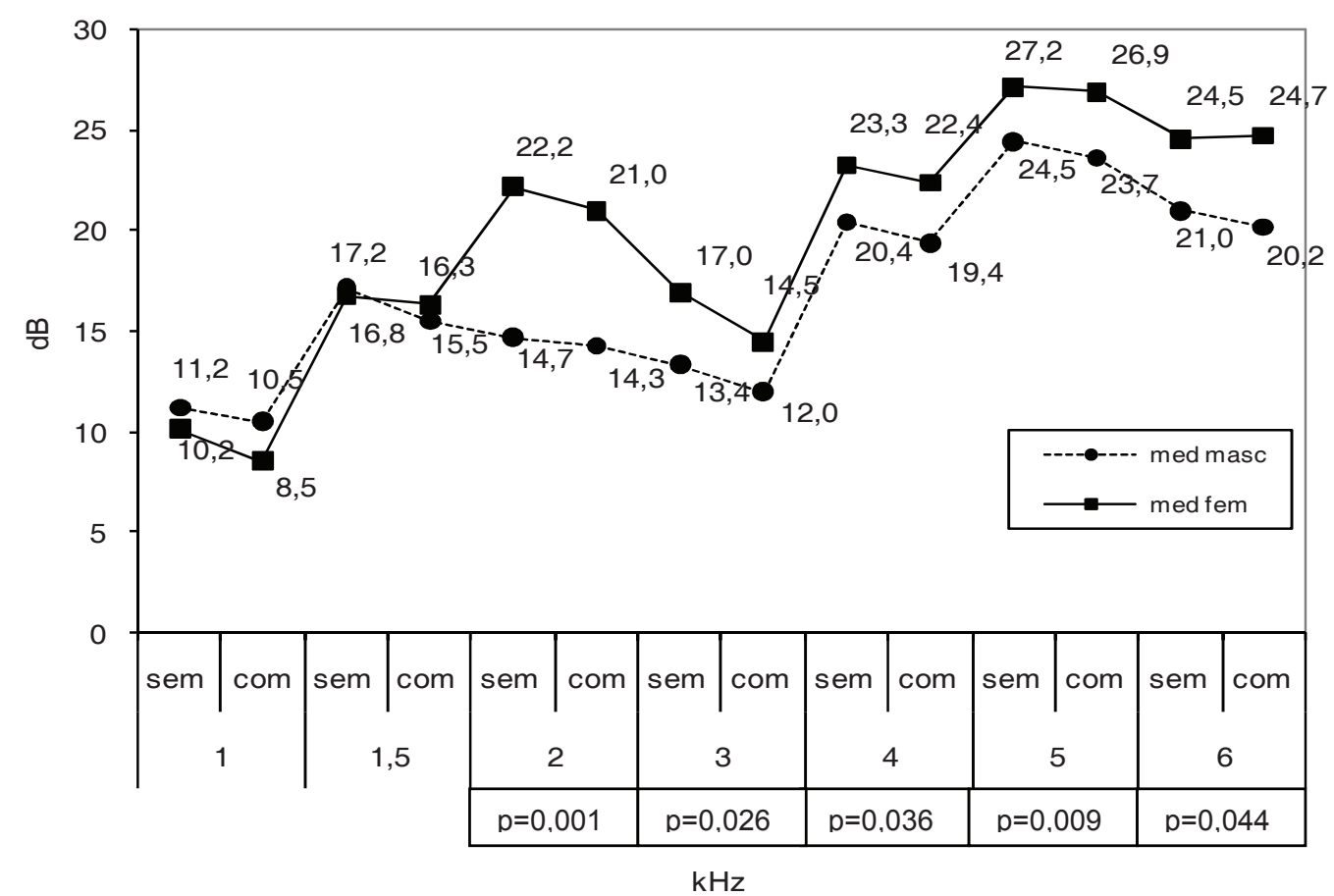

EOAPD: Emissões Otoacústicas Evocadas por produto de distorção; med: média; masc: maculino; fem: feminino; sem: sem ruído contralateral; com: com ruído contralateral.

Figura 1 - Comparação entre os sexos, da média dos níveis de respostas das EOAPD nas condições sem e com ruído contralateral 


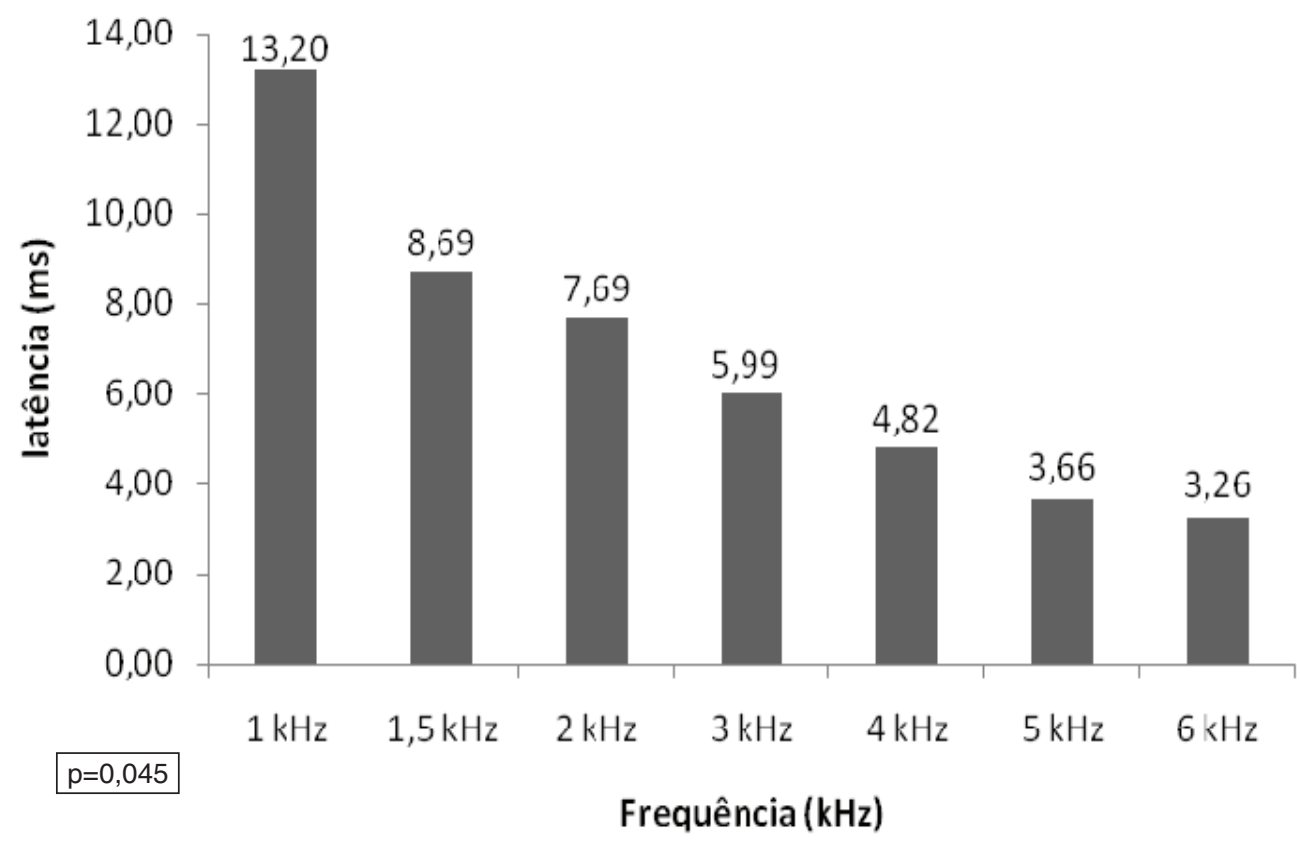

Figura 2 - Média dos valores de latência das EOAPD na condição sem ruído



Figura 3 - Distribuição dos percentis da diferença (condição sem ruído menos condição com ruído contralateral) para latência (A) e nível de resposta (B) das EOAPD nas frequências de $1 \mathrm{a} 6 \mathrm{kHz}$

Analisando-se a correlação entre nível de resposta e latência das EOAPD, os resultados demonstraram que os valores de supressão dos níveis de resposta das EOAPD não possuem nenhuma relação estatisticamente significante com os valores de latência das EOAPD.

\section{DISCUSSÃO}

No presente estudo não foi encontrada diferença estatisticamente significante nos níveis de resposta das EOAPD, entre os lados de orelha, na maioria das frequências pesquisadas, exceto em $1 \mathrm{kHz}$, 
em que a orelha direita apresentou maiores níveis de resposta em comparação à orelha esquerda na situação sem ruído e no efeito de supressão das EOAPD.

Alguns estudos descritos na literatura 13-15 encontraram níveis de respostas significantemente maiores na orelha direita, sendo que este achado tem sido relacionado aos mecanismos envolvendo a assimetria aural nas EOA espontâneas e à assimetria morfológica entre as regiões cranio-faciais direita e esquerda.

Quanto à latência de resposta, não se observou diferença estatisticamente significante entre os lados das orelhas, exceto na frequência de $5 \mathrm{kHz}$ na situação sem ruído em que a orelha esquerda apresentou maior valor de latência. Este resultado concorda com a literatura 5,16,17 que não referem efeito de orelha na latência das EOAPD tanto em neonatos como em adultos com audição normal.

Quanto à comparação dos resultados entre os gêneros, esta pesquisa concorda com estudo das EOA em neonatos ${ }^{18}$, que referem níveis de respostas das EOA maiores no sexo feminino e valores de latências maiores no sexo masculino, sendo este fato atribuído às diferenças morfométricas no comprimento coclear entre os gêneros, apontando comprimento coclear maior no sexo masculino ${ }^{19} \mathrm{e}$ também às diferenças do número de células ciliadas externas, descrito como mais numerosas no sexo feminino. Em estudo anterior sobre a latência das EOAPD ${ }^{17}$, não foi encontrada diferença estatisticamente significante nos valores de latência das EOAPD entre os sexos, apesar dos valores de latência no sexo masculino ter sido, em média, $6,35 \%$ maiores que no feminino.

Os resultados desta pesquisa demonstraram que os valores de latência das EOAPD diminuíram significantemente com o aumento da frequência sonora analisada, assim como no estudo sobre medidas de latência das emissões otoacústicas em neonatos ${ }^{5}$ e em adultos normo-ouvintes ${ }^{17}$, nos quais também se observaram diminuição significante nos valores da latência com o aumento da frequência sonora para ambos os sexos e para ambas as orelhas.

Este achado, portanto, concorda com a literatura e é relacionado com a tonotopia coclear, sendo que os sons de altas frequências estimulam a base da cóclea enquanto os sons de baixa frequência estimulam regiões do ápice da cóclea. Desta forma, sugere-se que a latência da resposta aumenta nas frequências baixas porque o som percorre uma distância maior ao longo da cóclea ${ }^{20}$.

Observou-se nesta pesquisa que houve diminuição significante dos níveis de magnitude de resposta das EOAPD com a apresentação de estimulação acústica contralateral. Desta forma, o ruído contralateral produziu o efeito de supressão das emissões otoacústicas, relacionado com a ação do sistema auditivo eferente, concordando com dados já relatados por inúmeros autores ${ }^{7-13}$.

Quanto ao objetivo principal deste trabalho, ou seja, investigar o efeito da estimulação acústica contralateral nas medidas temporais das EOAPD, os resultados não demonstraram diferença estatisticamente significante nos valores de latência das EOAPD comparando-se as condições sem e com ruído contralateral. Desta forma, no presente estudo não foi observado efeito da estimulação acústica contralateral, modificando significantemente valores de latência das EOAPD.

A presente pesquisa concorda com o estudo de pesquisadores chineses ${ }^{21}$ que investigaram o efeito da atenção seletiva e da estimulação acústica contralateral na latência das EOAPD. Foram analisados os valores de latência das EOAPD, em quinze sujeitos com audição normal, em três diferentes situações: com e sem tarefa de atenção visual, estimulação acústica contralateral e uma combinação das duas formas de estimulação. Os resultados não demonstraram diferença significante na latência das EOAPD nas três formas de estimulação, sendo que os autores concluíram que o efeito da atenção seletiva e da estimulação acústica contralateral na latência das EOA ainda deve ser mais estudado.

Contudo, em estudo sobre a supressão contralateral da latência durante a detecção das EOAPD em porcos da índia ${ }^{22}$, foram medidas amplitude e latência das EOAPD em 12 orelhas de porcos da índia saudáveis, com e sem a apresentação contralateral de ruído de banda larga com intensidade de 70 dB NPS; sendo observado prolongamento significante da latência das EOAPD durante a estimulação acústica contralateral. Os autores concluíram que a latência é um índice sensível na supressão contralateral das EOAPD em animais.

Apesar do fenômeno da supressão do nível de magnitude de resposta das EOA com a apresentação de ruído contralateral já ter sido bastante investigado e descrito por diversos autores, ainda é escassa a literatura sobre o efeito da ativação do sistema eferente, por meio de estimulação acústica contralateral, nas medidas de tempo de latência das EOAPD. A realização de novos estudos sobre este tema, utilizando-se metodologia rigorosa e que possa ser reproduzida, irá contribuir na investigação sobre as funções do sistema auditivo eferente.

\section{CONCLUSÃO}

Neste estudo, não houve diferença estatisticamente significante nos valores de latência das EOAPD de adultos com a audição normal, 
comparando-se as condições sem e com a apresentação de ruído contralateral.

Observou-se que os valores de latência das EOAPD diminuíram significantemente com o aumento da frequência sonora analisada.

Não foi observada diferença estatisticamente significante nos valores de nível de resposta e de latência das EOAPD, comparando-se os lados de orelha.

Quanto à influência do sexo, observou-se níveis de respostas das EOAPD maiores no sexo feminino e valores de latência maiores no sexo masculino na condição sem ruído. Contudo, não foi observada diferença estatisticamente significante entre os sexos, tanto para nível de resposta como para latência, no efeito de supressão das EOAPD.

Desta forma, os resultados deste estudo permitem concluir que o sistema auditivo eferente atuou apenas na modulação dos níveis de magnitude de resposta das EOAPD, contudo não interferiu nas medidas temporais das mesmas.

\begin{abstract}
Purpose: compare the otoacoustic emissions response level and latency in two situations: with and without contralateral competitive noise. Methods: thirty individuals between eighteen and thirty-year-old, with no auditory complaints and presence of otoacoustic distortion product emissions were evaluated. The latency and the response level of otoacoustic emissions was evaluated using the Latencygram program. The test was performed in two different situations: with and without contralateral competitive white noise. Results: the results demonstrated a significant reduction in otoacoustic emissions response levels with contralateral noise, proving the otoacoustic suppression effect associated with olivocochlear efferent system action. However, there was no significant difference in otoacoustic emissions latency. Conclusion: the efferent system may act only in otoacoustic emissions response level modulation but it does not interfere in otoacoustic emissions latency.
\end{abstract}

KEYWORDS: Otoacoustic Emissions, Spontaneous; Reaction Time; Acoustic Estimulation

\section{REFERÊNCIAS}

1. Abissamra RGC. Latências das emissões otoacústicas da orelha normal de portadores de perda auditiva sensorioneural unilateral. [dissertação] São Paulo (SP): Universidade de São Paulo; 2001.

2. Neely ST, Johnson TA, Gorga MP. Distortionproduct otoacoustic emission measured with continuously varying stimulus level. J Acoust Soc Am. 2005; 117(3Pt1):1248-59.

3. Shaffer LA, Withnell RH, Dhar S, Lilly DJ, Goodman SS, Harmon KM. Sources and mechanisms of DPOAE generation: implications for the prediction of auditory sensitivity. Ear Hear. 2003; 24(5):367-79.

4. Quiñonez RE, Crawford MR. Longitudinal distortion product otoacoustic emission (DPE) latency changes in preterms neonates. Acta Otolaryngol. 1998; 118(1):26-31.

5. Azevedo RF, Carvallo RMM. Medidas da latência das emissões otoacústicas - produto de distorção em neonatos. Rev Bras Otorrinolaringol. 2003; 69(5):691-5.

6. May BJ, Budelis J, Niparko JK. Behavioral studies of the olivocochlear efferent system: learning to listen in noise. Arch Otolaryngol Head Neck Surg. 2004; 130(5):660-4.
7. Guinan Junior JJ. Olivocochlear efferents: anatomy, physiology, function, and the measurement of efferent effects in humans. Ear Hear. 2006; 27(6):589-607.

8. Fávero ML, Sanchez TG, Nascimento AF, Bento RF. A função do trato olivococlear medial em indivíduos com zumbido. Arq Otorrinolaringol. 2003; 7(4):265-70.

9. Groff JA, Liberman MC. Modulation of cochlear afferent response by the lateral olivocochlear system: activation via electrical stimulation of the inferior colliculus. J Neurophysiol. 2003; 90(5):3178-200.

10. Muchnik C, Roth DA, Othman-Jebara R, PutterKatz H, Shabtai EL, Hildesheimer M. Reduced medial olivocochlear bundle system function in children with auditory processing disorders. Audiol Neurootol. 2004; 9(2):107-14.

11. Mor R, Azevedo MF. Emissões otoacústicas e sistema olivococlear medial: pacientes com zumbido sem perda auditiva. Pró-Fono. 2005; 17(3):283-92.

12. Sanches SGG, Carvallo RM. Contralateral supression of transient evoked otoacoustic emissions in children with auditory processing disorder. Audiol Neurootol. 2006; 11(6):366-72. 
13. Durante AS, Carvallo RMM. Contralateral suppression of linear and nonlinear transient evoked otoacoustic emissions in neonates at risk for hearing loss. J Commun Disord. 2008; 41(1):70-83.

14. Kumar UA, Vanaja CS. Functioning of olivocochlear bundle and speech perception in noise. Ear Hear. 2004; 25(2):142-6.

15. Tadros SF, Frisina ST, Mapes F, Kim S, Frisina DR, Frisina RD. Loss of peripheral rightear advantage in age-related hearing loss. Audiol Neurootol. 2005; 10(1):44-52.

16. Marques VV, Azevedo MF. Distortion product emission latency in normal hearing adults. PróFono. 2004; 16(2):203-8.

17. Campos UP, Carvallo RMM. Latência das EOAPD em milissegundos e número de ondas. Rev Bras Otorrinolaringol. 2005; 71(6):784-90.

18. Basseto MCA, Chiari BM, Azevedo MF. Emissões otoacústicas evocadas transientes (EOAET): amplitude de resposta em recém-nascidos a termo e pré-termo. Rev Bras Otorrinolaringol. 2003; 69(1):84-92.

19. Bowman DM, Brown DK, Kimberley BP. An examination of gender differences in DPOAE phase delay measurements in normal-hearing human adults. Hear Res. 2000; 142(1-2):1-11.

20. Mahoney CF, Kemp DT. Distortion product otoacoustic emission delay measurement in human ears. J Acoust Soc Am. 1995; 97(6):3721-35.

21. Zhong N, Li Q, Guo Y. [Effects of selective attention and contralateral acoustic stimulation on latency of distortion product otoacoustic emissions]. Lin Chuang $\mathrm{Er} B \mathrm{Bi}$ Yan Hou Ke Za Zhi. 2001; 15(10):437-8.

22. Kong W, Yang $Y$, Zhang W. [Contralateral suppression of latency during distortion product otoacoustic emissions detection in guinea pigs]. Zhonghua $\mathrm{Er} \mathrm{Bi}$ Yan Hou Ke Za Zhi. 2001; 36(4):271-4
DOI: 10.1590 / S1516- 18462009005000008

RECEBIDO EM: 28/01/2008

ACEITO EM: 13/07/2008

Endereço para correspondência:

Vanessa Nogueira Leme

Rua 28 de setembro, 490 ap.15

Caçapava - SP

CEP: $12282-900$

E-mail: vanessafonousp@yahoo.com.br 